\title{
Some Comments on the Sherrington-Kirkpatrick Model of Spin Glasses
}

\author{
J. Fröhlich ${ }^{1, \star}$ and B. Zegarlinski ${ }^{2}$ \\ ${ }^{1}$ I.H.E.S., 35 Route de Chartres, F-91440 Bures-sur-Yvette, France \\ ${ }^{2}$ Institute of Theoretical Physics, University of Wrocław, Wrocław, Poland
}

\begin{abstract}
In this paper the high-temperature phase of general mean-field spin glass models, including the Sherrington-Kirkpatrick (SK) model, is analyzed. The free energy in zero magnetic field is calculated explicitly for the SK model, and uniform bounds on quenched susceptibilities are established. It is also shown that, at high temperatures, mean-field spin glasses are limits of shortrange spin glasses, as the range of the interactions tends to infinity.
\end{abstract}

\section{Introduction}

In this note we comment on the high-temperature properties of a class of meanfield spin glass models, including the Sherrington-Kirkpatrick (SK) model. Our method of analysis is the one developed in [1]. In [2], Aizenman et al. present a detailed analysis of the high-temperature behaviour of the SK model in zero magnetic field ${ }^{1}$. They also prove some results on the behaviour of the free energy at low temperatures and give bounds on the ground state energy density. While their results prove that the SK model exhibits a phase transition in zero magnetic field, as the temperature is lowered, a lot of work remains to be done to show that the Parisi replica symmetry breaking solution [3] of the SK model is exact or, at least, qualitatively correct. There is, however, a simpler mean-field type model, the Ising spin glass on a Bethe tree [4] for which Chayes, Chayes, Sethna and Thouless have been able to perform a rather complete analysis [5]. Their conclusions which are mathematically rigorous are qualitatively similar to those obtained in the Parisi solution. Heuristic analyses of the short-range Edwards-Anderson spin glass [6] have been carried out in [7-9]. The picture that emerges is still somewhat controversial, but some of the main features of the low-temperature phase of the short-range Ising spin glass on a sufficiently high-dimensional lattice appear to be reminiscent of the Parisi solution $[7,8]$. (For example, the space of equilibrium states, at small $T$ and $h=0$, appears to exhibit an ultrametric structure [8].)

\footnotetext{
^ Permanent address: Theoretical Physics, ETH-Hönggerberg, CH-8093 Zürich, Switzerland

${ }^{1}$ We thank D. Ruelle for providing us with a copy of [2] prior to publication
} 
The point of our note is to show that the simple and rather generally applicable methods developed in [1] suffice to understand the main properties of a class of mean-field spin glass models of general $n$-vector spins $(n=1,2,3, \ldots ; n=1$ corresponds to Ising spins) in an arbitrary magnetic field $h$, including the SK model, provided the temperature is large enough. The analytic expression for the free energy of the SK model at high temperature and in zero magnetic field, obtained in [10] and rigorously established in [2] and in this paper, cannot be correct at sufficiently low temperature, since it would violate the positivity of the entropy. Therefore, in zero magnetic field, the SK model exhibits a phase transition, as the temperature is lowered. This point is discussed in more detail in [2].

For simplicity, we shall prove our results for Ising spins only, but the extension to general $n$-vector spins is straightforward; see the second ref. quoted in [1].

\section{Notations and Main Results}

We consider an array of $N$ points, $j=1, \ldots, N$, carrying a $\operatorname{spin} \mathbf{S}_{j}, \mathbf{S}_{j}=\left(S_{j}^{1}, \ldots, S_{j}^{n}\right)$, with $\left|\mathbf{S}_{j}\right|=1$. The energy of a configuration $\left\{\mathbf{S}_{j}\right\}$ is given by a Hamilton function

$$
H_{N}=-\sum_{1 \leqq i<j \leqq N}\left[\frac{J_{i j}}{\sqrt{N}}+\frac{J^{0}}{N}\right] g\left(\mathbf{S}_{i}, \mathbf{S}_{j}\right)-h \sum_{1 \leqq i \leqq N} S_{i}^{1},
$$

where $g\left(\mathbf{S}_{i}, \mathbf{S}_{j}\right)$ is a bounded continuous function on the product space $S_{n-1} \times S_{n-1}$ of configurations of $\mathbf{S}_{i}$ and $\mathbf{S}_{j}$. The precise choice of $g$ is not important, but in our proofs we shall, for simplicity, set

$$
g\left(\mathbf{S}, \mathbf{S}^{\prime}\right)=\mathbf{S} \cdot \mathbf{S}^{\prime}
$$

(Different choices of $g$ are significant at low temperatures, but are quite immaterial at high temperatures.) The exchange coupling $J^{0}$ is fixed, while the $J_{i j}$ 's are independent, identically distributed random variables. Expectations in $\left\{J_{i j}\right\}$ are denoted by $E$, and it is assumed that

$$
E J_{i j}=0, \quad E J_{i j}^{2}=J^{2}>0, \quad E J_{i j}^{2 p} \leqq(c J)^{2 p}(2 p) !, \quad p>1,
$$

for some finite constant $c$. For simplicity, we also assume that $E$ is even, but this is unimportant. When $E$ is the $\delta$-distribution concentrated at $J_{i j}=0$, for all (ij) (= unordered pairs of sites, with $i \neq j$ ) the Hamiltonian is denoted by $H_{N}^{0}$. This is the usual ferromagnetic $\left(J^{0}>0\right)$ or anti-ferromagnetic $\left(J^{0}<0\right)$ mean-field Hamiltonian. The standard spin glass Hamiltonian corresponds to the choice $J^{0}=0$, but spin glass behaviour may be observed at low temperatures even when $\left|J^{0}\right|>0$ is sufficiently small.

The free energy density, $f$, of the system is defined by

$$
\beta f\left(\beta H_{N}\right)=-\frac{1}{N} \log \mu^{0}\left(e^{-\beta H_{N}}\right),
$$

where $\mu^{0}$ is the product measure on $\left(S_{n-1}\right)^{\times N}$ coinciding with the uniform measure on each factor. In particular, for Ising spins, $\mu^{0}=2^{-N} \times$ counting measure. 
Let $\mu_{N}$ be the equilibrium state given by

$$
\mu_{N}(\cdot)=\left[\mu^{0}\left(e^{-\beta H_{N}}\right)\right]^{-1} \mu^{0}\left(e^{-\beta H_{N}} \cdot\right) .
$$

We introduce the susceptibilities

$$
\begin{gathered}
\chi_{N}^{(1)}(\beta, h)=N^{-1} \sum_{1 \leqq i<j \leqq N}\left|E \mu_{N}\left(\mathbf{S}_{i} \cdot \mathbf{S}_{j}\right)^{c}\right|, \\
\chi_{N}^{(2)}(\beta, h)=N^{-1} \sum_{1 \leqq i<j \leqq N} E\left(\mu_{N}\left(\mathbf{S}_{i} \cdot \mathbf{S}_{j}\right)^{c}\right)^{2},
\end{gathered}
$$

where

$$
\mu_{N}\left(\mathbf{S}_{i} \cdot \mathbf{S}_{j}\right)^{c}=\mu_{N}\left(\mathbf{S}_{i} \cdot \mathbf{S}_{j}\right)-\mu_{N}\left(\mathbf{S}_{i}\right) \cdot \mu_{N}\left(\mathbf{S}_{j}\right)
$$

Let $f\left(\beta H_{N}^{0}\right)$ be the usual mean-field free energy for the deterministic model, $\left[J_{i j}=0\right.$, for all $\left.(i j)\right]$. We are now prepared to state our main result.

Theorem A. (1) For all $n=1,2,3, \ldots$ and arbitrary $\beta$ and $h$,

$$
f\left(\beta H_{N}^{0}\right) \geqq E f\left(\beta H_{N}\right) \geqq f\left(\beta H_{N}^{0}\right)-\frac{\beta J^{2}}{4} .
$$

(2) For all $n=1,2,3, \ldots$, arbitrary $h$ and $\beta$ small enough,

$$
\chi_{N}^{(i)}(\beta, h) \leqq C^{(i)}<\infty, \quad i=1,2,
$$

uniformly in $N$.

(3) For Ising spins $(n=1)$, with $h=0$ and $\beta$ small enough,

$$
\begin{gathered}
E f\left(\beta H_{N}\right)=f\left(\beta H_{N}^{0}\right)-\frac{\beta J^{2}}{4}+O\left(\frac{1}{N}\right), \\
E\left(f\left(\beta H_{N}\right)-E f\left(\beta H_{N}\right)\right)^{2}=O\left(\frac{1}{N^{2}}\right) .
\end{gathered}
$$

It is natural to ask whether the SK model may be obtained as the infinite range limit of short range spin glasses on a finite-dimensional lattice. That such a result ought to be true must have been part of the original motivation to introduce the SK model. Thus we consider a spin glass on the $d$-dimensional hypercubic lattice $\mathbb{Z}^{d}$ with Hamilton function

$$
H_{\Lambda}^{\gamma}=-\sum_{(i j) \subset A} J_{i j}^{\gamma} \mathbf{S}_{i} \cdot \mathbf{S}_{j}-h \sum_{i \in \Lambda} S_{i}^{1}
$$

where

$$
J_{i j}^{\gamma}=\left(\mathscr{J}_{i j} \gamma^{d / 2}+\widetilde{J}^{0} \gamma^{d}\right) \exp [-\gamma|i-j|],
$$

the $\mathscr{J}_{i j}$ 's are independent, identically distributed random variables with expectation $\widetilde{E}$ satisfying $(2.2)$, for some constants $\widetilde{J}$ and $\tilde{c}$. Let $f_{\Lambda}(\beta, h, \gamma)$ be the free energy and $\mu_{A}^{\gamma}$ the finite-volume equilibrium state of the model. In [1] we have shown that, for $\beta$ small enough, the thermodynamic limits of these quantities exist and are unique, for all $\gamma>0$; (for precise statements see [1]).

Our second main result is the following theorem. 
Theorem B. For Ising spins $(n=1)$, and for $h=0, \beta$ small enough, and for a suitable choice of $\widetilde{J}^{0}$ and $\widetilde{E}$ (given $J^{0}$ and $E$ ),

$$
\lim _{\gamma>0}\left(\lim _{\Lambda \rightarrow \mathbb{Z}^{d}} f_{\Lambda}(\beta, 0, \gamma)\right)=\lim _{N \rightarrow \infty} f\left(\beta H_{N}\right)=\lim _{N \rightarrow \infty} f\left(\beta H_{N}^{0}\right)-\frac{\beta J^{2}}{4} .
$$

( The limits $\Lambda \nearrow \mathbb{Z}^{d}, N \rightarrow \infty$ are understood in the sense of $L_{p}$, with respect to $E, \widetilde{E}$, respectively, $1 \leqq p<\infty$.)

Remark. This result is a special case of more general results relating thermodynamic and correlation functions for the short-range models to the corresponding quantities for the mean-field model, in the limit when $\gamma \searrow 0$; see [11].

\section{Proofs of Main Results}

The proofs of Theorems A and B are straightforward adaptations of the arguments in [1]. (While we feel the first paper quoted in [1] shows how simple and general our techniques are, that simplicity was somewhat obscured in the second paper of [1], because of a certain overemphasis of generality. In order not to fall into the same trap, we here prove our main results in the simplest cases. The reader familiar with [1] will easily understand how to recover the general case, but see also [11].)

Let

$$
\varphi\left(\beta H_{N}\right)=\frac{1}{N} \log \mu^{0}\left(e^{-\beta H_{N}}\right) .
$$

By Jensen's inequality $\varphi\left(\beta H_{N}\right) \geqq 0$. Let $\mu_{N}^{0}$ be the equilibrium state associated with $H_{N}^{0}$, and define $\delta H_{N}$ by

$$
\delta H_{N}=-\sum_{1 \leqq i<j \leqq N} \frac{J_{i j}}{\sqrt{N}} \mathbf{S}_{i} \cdot \mathbf{S}_{j}
$$

By Jensen's inequality

$$
\varphi\left(\beta H_{N}\right) \geqq \varphi\left(\beta H_{N}^{0}\right)-\frac{\beta}{N^{3 / 2}} \sum_{1 \leqq i<j \leqq N} J_{i j} \mu_{N}^{0}\left(\mathbf{S}_{i} \cdot \mathbf{S}_{j}\right) .
$$

Hence, by (2.2)

$$
E \varphi\left(\beta H_{N}\right) \geqq \varphi\left(\beta H_{N}^{0}\right)
$$

Since

$$
\varphi\left(\beta H_{N}\right)=-\beta f\left(\beta H_{N}\right),
$$

this proves the upper bound in Theorem A, (1). [We shall see that, as $N \rightarrow \infty$, the expectation $E$ may be omitted, by the self-averaging property of $\lim _{N \rightarrow \infty} f\left(\beta H_{N}\right)$, for $\beta$ small enough.]

In order to prove the lower bound in Theorem A, (1) we apply a Taylor expansion in $\delta H_{N}$ with second order remainder. We order the pairs $(i j)$ 
lexicographically and first expand in $\frac{J_{12}}{\sqrt{N}} \mathbf{S}_{1} \cdot \mathbf{S}_{2}$. This yields

$$
\begin{aligned}
\varphi\left(\beta H_{N}\right)= & \left.\varphi\left(\beta \mathrm{H}_{N}\right)\right|_{J_{12}=0}+\left.\frac{\beta}{N^{3 / 2}} J_{12} \mu_{N}\left(\mathbf{S}_{1} \cdot \mathbf{S}_{2}\right)\right|_{J_{12}=0} \\
& +\left.\frac{\beta^{2}}{N^{2}} \int_{0}^{1} d s_{12} \int_{0}^{s_{12}} d s_{12}^{\prime} J_{12}^{2} \mu_{N}\left(\mathbf{S}_{1} \cdot \mathbf{S}_{2} ; \mathbf{S}_{1} \cdot \mathbf{S}_{2}\right)\right|_{S_{12}^{\prime}},
\end{aligned}
$$

where

$$
\mu_{N}(A ; B)=\mu_{N}(A \cdot B)-\mu_{N}(A) \mu_{N}(B), \quad S_{i j}=\beta s_{i j} J_{i j}, \quad S_{i j}^{\prime}=\beta s_{i j}^{\prime} J_{i j}, \ldots,
$$

and

$$
\left.\mu_{N}(F)^{p}\right|_{J_{A, B}=0, S_{C, D}}
$$

indicates the $p^{\text {th }}$ power of the expectation of a function $F$ of $\left\{\mathbf{S}_{i}\right\}_{i=1}^{N}$ in the state $\mu_{N}$ defined in (2.4), but with $J_{i j}$ replaced by zero, for all $i \in A, j \in B$, and with $\beta J_{i j}$ replaced by $S_{i j}$, for all $i \in C$ and all $j \in D$. This notation is extended to arbitrary sums and products of expectations in $\mu_{N}$.

We now iterate (3.5) by expanding $\left.\varphi\left(\beta H_{N}\right)\right|_{J_{12}=0}$ in $J_{13}$ to second order, etc. This yields

$$
\begin{aligned}
\varphi\left(\beta H_{N}\right)= & \varphi\left(\beta H_{N}^{0}\right)+\left.\frac{\beta}{N^{3 / 2}} \sum_{(i j)} J_{i j} \mu_{N}\left(\mathbf{S}_{i} \cdot \mathbf{S}_{j}\right)\right|_{C_{i j}} \\
& +\left.\frac{\beta^{2}}{N^{2}} \sum_{(i j)} \int_{0}^{1} d s_{i j} \int_{0}^{s_{2 j}} d s_{i j}^{\prime} J_{i j}^{2} \mu_{N}\left(\mathbf{S}_{i} \cdot \mathbf{S}_{j} ; \mathbf{S}_{i} \cdot \mathbf{S}_{j}\right)\right|_{C_{i j}^{\prime}},
\end{aligned}
$$

where $C_{i j}$ is the constraint $J_{i^{\prime} j^{\prime}}=0$, for all $\left(i^{\prime} j^{\prime}\right) \preceq(i j), \prec$ indicates the lexicographic order relation, and $C_{i j}^{\prime}$ is the constraint $J_{i^{\prime} j^{\prime}}=0$, for all $\left(i^{\prime} j^{\prime}\right) \prec(i j)$, and $J_{i j}$ is replaced by $s_{i j}^{\prime} J_{i j}$.

Taking expectations in $E$ on both sides of (3.7), we obtain by using (2.2)

$$
E \varphi\left(\beta H_{N}\right)=\varphi\left(\beta H_{N}^{0}\right)+\left.\frac{\beta^{2}}{N^{2}} \sum_{(i j)} \int_{0}^{1} d s_{i j} \int_{0}^{s_{i j}} d s_{i j}^{\prime} E J_{i j}^{2} \mu_{N}\left(\mathbf{S}_{i} \cdot \mathbf{S}_{j} ; \mathbf{S}_{i} \cdot \mathbf{S}_{j}\right)\right|_{C_{i_{j}^{\prime}}} .
$$

Since

$$
\mu_{N}\left(\left(\mathbf{S}_{i} \cdot \mathbf{S}_{j}\right)^{2}\right) \leqq 1
$$

we obtain from (3.8) by simple calculations

$$
E \varphi\left(\beta H_{N}\right) \leqq \varphi\left(\beta H_{N}^{0}\right)+\frac{\beta^{2} J^{2}}{4}\left(1-\frac{1}{N}\right),
$$

which completes the proof of Theorem A, (1).

The methods in [1] can be used to derive more detailed information on the right-hand side of (3.8). [For this purpose one continues the expansion of the second term on the right-hand side of (3.8) in $\left\{J_{k l}\right\}$. $]$ Here we just note that, for Ising spins $(n=1)$,

$$
\left(\mathbf{S}_{i} \cdot \mathbf{S}_{j}\right)^{2}=1
$$


and we obtain from (3.8),

$$
E \varphi\left(\beta H_{N}\right)=\varphi\left(\beta H_{N}^{0}\right)+\frac{\beta^{2} J^{2}}{4}\left(1-\frac{1}{N}\right)-\frac{\beta^{2}}{N^{2}} \sum_{(i j)} \int_{0}^{1} d s_{i j} \int_{0}^{s_{i j}} d s_{i j}^{\prime} E J_{i j}^{2}\left(\left.\mu_{N}\left(\mathbf{S}_{i} \cdot \mathbf{S}_{j}\right)\right|_{C_{i j}^{\prime}}\right)^{2} .
$$

In order to prove Theorem A, (3) and a part of Theorem A, (2) $(h=0$ and $i=2)$, it is enough to prove a uniform upper bound on

$$
N^{-1} \sum_{(i j)} E \lambda_{i j}(J)\left(\left.\mu_{N}\left(\mathbf{S}_{i} \cdot \mathbf{S}_{j}\right)\right|_{\tilde{c}_{i j}}\right)^{2}
$$

where, for all $(i j), \lambda_{i j}(J)$ is an even, positive function of $\left\{J_{k l}\right\}$ with the properties $E \lambda_{i j}(J) \leqq L, E \lambda_{i j}(J) J_{k l}=0$ and $E \lambda_{i j}(J) J_{k l}^{2 p} \leqq\left(c^{\prime} J\right)^{2 p}(2 p)$ !, for all $p \geqq 1$, with $L$ and $c^{\prime}$ independent of $(i j)$ and $(k l)$. If the exchange couplings compatible with the constraint $\widetilde{C}_{i j}$ are denoted by $\widetilde{J}_{m n}$, then

$$
\tilde{J}_{m n}=a(m n, i j) J_{m n},
$$

with $a(m n, i j) \in[0,1]$, for each $(m n)$. We set

$$
I_{N}^{(2)}(i, j)=\left(\left.\mu_{N}\left(\mathbf{S}_{i} \cdot \mathbf{S}_{j}\right)^{c}\right|_{\tilde{c}_{i j}}\right)^{2}
$$

and

$$
E_{\lambda}(\cdot) \equiv E_{\lambda_{i j}}(\cdot)=E\left(\lambda_{i j}(J) \cdot\right) .
$$

We propose to prove now that, for $\beta$ small enough,

$$
N^{-1} \sum_{(i j)} E_{\lambda_{\imath J}} I_{N}^{(2)}(i, j) \leqq C^{(2)}<\infty,
$$

uniformly in $N$. For simplicity, we present a proof for Ising spins $(n=1)$ and $h=0$ only, but the general case is handled by combining the present arguments with the techniques of Sect. 3 of the second paper quoted in [1].

Let $K_{i j}=\frac{J^{0}}{N}+\frac{\widetilde{J}_{i j}}{\sqrt{N}}$ be the total exchange coupling constant between spins $\mathbf{S}_{i}$ and $\mathbf{S}_{j}$, and let $S_{i j}=\beta s_{i j} K_{i j}, S_{i j}^{\prime}=\beta s_{i j}^{\prime} K_{i j}$. Let us denote a derivative with respect to $S_{i j}^{(\prime)}$ by $\partial_{i j}$. By a Taylor expansion in $K_{i j}$ with remainder we obtain

$$
\begin{aligned}
E_{\lambda} I_{N}^{(2)}(i, j)= & \left.E_{\lambda} I_{N}^{(2)}(i, j)\right|_{K_{\imath j}=0}+\left.\beta E_{\lambda}\left(\frac{J^{0}}{N}+\frac{\tilde{J}_{i j}}{\sqrt{N}}\right) \partial_{i j} I_{N}^{(2)}(i, j)\right|_{K_{\imath j}=0} \\
& +\left.\beta^{2} \int_{0}^{1} d s_{i j} \int_{0}^{s_{i j}} d s_{i j}^{\prime} E_{\lambda}\left(\frac{J^{0}}{N}+\frac{\tilde{J}_{i j}}{\sqrt{N}}\right)^{2} \partial_{i j}^{2} I_{N}^{(2)}(i, j)\right|_{\mathscr{C}_{\imath j}},
\end{aligned}
$$

where $\mathscr{C}_{i j}$ denotes the constraint which replaces $K_{i j}$ by $s_{i j}^{\prime} K_{i j}$. Since $E_{\lambda} \widetilde{J}_{i j}=0$, we conclude that

$$
E_{\lambda} I_{N}^{(2)}(i, j) \leqq\left. E_{\lambda} I_{N}^{(2)}(i, j)\right|_{K_{i j}=0}+\frac{\beta J^{0}}{N} a_{1}+\frac{(\beta J)^{2}}{N} a_{2}+O\left(\frac{1}{N^{2}}\right)
$$

with $\left|a_{1}\right| \leqq 2 E_{\lambda} 1,\left|a_{2}\right| \leqq 2 J^{-2} E_{\lambda} J_{i j}^{2}$. In passing from (3.15) to (3.16) one calculates $\left.\partial_{i j}^{\alpha} I_{N}^{(2)}(i, j)\right|_{\mathscr{C}_{\imath J}}, \alpha=1,2$, explicitly, using $\mathbf{S}_{i}^{2}=1$; (for Ising spins; in general $n$-vector models, one still gets a uniform upper bound on $\left|a_{i}\right|, i=1,2$ ). 
Next, we Taylor-expand the first term on the right-hand side of (3.16) in $K_{i k}$ to second order, where $k$ is the smallest site $\neq i, j$, then we expand the constant term in $K_{i k^{\prime}}$, with $k^{\prime}>k, k^{\prime} \neq i, j$, etc. (This is the method of [1].) This yields

$$
\begin{gathered}
\left.E_{\lambda} I_{N}^{(2)}(i, j)\right|_{K_{\imath j}=0}=\left.\beta \sum_{k \neq i, j} E_{\lambda}\left(\frac{J^{0}}{N}+\frac{\widetilde{J}_{i k}}{\sqrt{N}}\right) \partial_{i k} I_{N}^{(2)}(i, j)\right|_{C_{i j k}} \\
+\left.\beta^{2} \sum_{k \neq i, j} \int_{0}^{1} d s_{i k} \int_{0}^{s_{i k}} d s_{i k}^{\prime} E_{\lambda}\left(\frac{J^{0}}{N}+\frac{\widetilde{J}_{i k}}{\sqrt{N}}\right)^{2} \partial_{i k}^{2} I_{N}^{(2)}(i, j)\right|_{\mathscr{C}_{i j k}},
\end{gathered}
$$

where $C_{i j k}$ sets $K_{i j}=0$ and $K_{i k^{\prime}}=0$, for all $k^{\prime} \leqq k$, and $\mathscr{C}_{i j k}$ sets $K_{i j}=0$ and $K_{i k^{\prime}}=0$, for all $k^{\prime}<k$, and replaces $\beta K_{i k}$ by $S_{i k}^{\prime}$. The constant term, $\left.I_{N}^{(2)}(i, j)\right|_{K_{i k}=0, \forall k \neq i}$, on the right-hand side of (3.17) vanishes, since $\mu^{0}\left(\mathbf{S}_{i}\right)=0$. We also use that $E_{\lambda} \widetilde{J}_{i k}=0$. Hence (3.17) yields the bound

$$
\begin{aligned}
& \left.E_{\lambda} I_{N}^{(2)}(i, j)\right|_{K_{\imath j}=0} \leqq\left.\frac{\beta J^{0}}{N} \sum_{k \neq i, j} E_{\lambda} \partial_{i k} I_{N}^{(2)}(i, j)\right|_{C_{\imath \jmath k}} \\
& \quad+2 \frac{\beta^{2}}{N} \sum_{k \neq i, j} \int_{0}^{1} d s_{i k} \int_{0}^{s_{\imath k}} d s_{i k}^{\prime} E_{\lambda} \widetilde{J}_{i k}^{2}\left|\partial_{i k}^{2} I_{N}^{(2)}(i, j)\right|_{\mathscr{C}_{\imath j k}} \mid+\frac{20 \beta^{2}}{N}\left(J^{0}\right)^{2} E_{\lambda} 1 .
\end{aligned}
$$

Carrying out differentiations in $S_{i k}^{\prime}$ explicitly and using such sophisticated inequalities as $|a \cdot b| \leqq \frac{1}{2}\left(a^{2}+b^{2}\right)$, one shows that

$$
\left|\partial_{i k}^{\alpha} I_{N}^{(2)}(i, j)\right|_{C} \mid \leqq K\left[\left.I_{N}^{(2)}(i, j)\right|_{C}+\left.I_{N}^{(2)}(k, j)\right|_{C}\right],
$$

for $\alpha=1,2$. The condition $C$ is arbitrary and $K \leqq 12$. Inequalities (3.18) and (3.19) give

$$
\begin{aligned}
& \left.E_{\lambda} I_{N}^{(2)}(i, j)\right|_{K_{i j}=0} \leqq\left.\frac{\beta K J^{0}}{N} \sum_{k \neq i, j} E_{\lambda}\left(I_{N}^{(2)}(i, j)+I_{N}^{(2)}(k, j)\right)\right|_{C_{\imath j k}} \\
& \quad+\left.\frac{2 \beta^{2} K}{N} \sum_{k \neq i, j} \int_{0}^{1} d s_{i k} \int_{0}^{s_{i k}} d s_{i k}^{\prime} E_{\lambda} \widetilde{J}_{i k}^{2}\left(I_{N}^{(2)}(i, j)+I_{N}^{(2)}(k, j)\right)\right|_{\mathscr{C}_{i j k}}+\frac{20 \beta^{2}}{N}\left(J^{0}\right)^{2} E_{\lambda} 1 .
\end{aligned}
$$

The bound (3.20) can now be iterated by replacing the terms proportional to $\left.I_{N}^{(2)}(i, j)\right|_{C_{i j k}}$ and to $\left.I_{N}^{(2)}(i, j)\right|_{\mathscr{C}_{i j k}}$ on the right-hand side of (3.20) by upper bounds similar to (3.20), and so on. In order to estimate the resulting expansion, we combine property (2.2) of $E$ with the trivial fact that

$$
\int_{0}^{1} d s \int_{0}^{s} d s^{(1)} \cdots \int_{0}^{s^{(n-1)}} d s^{(n)} a=\frac{a}{(n+1) !} .
$$

More specifically, we define

$$
\bar{I}_{N}^{(2)}(k, l)=\sup _{C, p} \bar{I}_{N}^{(2)}(k, l ; C, p)
$$

where

$$
\begin{aligned}
\bar{I}_{N}^{(2)}(k, l ; C, p)=\prod_{(m n)}\left\{\int_{0}^{1}\right. & \left.d s_{m n}^{[1]} \ldots \int_{0}^{s\left[p_{n}(m n)-1\right]} d s_{m n}^{[p(m n)]}\right\} \\
& \times E_{\lambda}\left(\left.\prod_{(m n)}\left(\beta \widetilde{J}_{m n}\right)^{p(m n)} \mu_{N}\left(\mathbf{S}_{k} \cdot \mathbf{S}_{l}\right)^{c}\right|_{C, S[p]}\right)
\end{aligned}
$$


Here $p(m n)=0,2,4, \ldots$, for all $(m n), C$ is an arbitrary constraint replacing $K_{m n}$ by $K_{m n}^{C}=a(m n, C) K_{m n}$, with $a(m n, C) \in[0,1]$, for all $(m n)$, and $S^{[p]}$ indicates that $\beta K_{m n}^{C}$ is replaced by $S_{m n}^{[p(m n)]}=\beta S_{m n}^{[p(m n)]} K_{m n}^{C}$. The steps from (3.15) to (3.20) can be repeated for every $\bar{I}_{N}^{2)}(k, l ; C, p)$, and (3.20) can then be iterated, as announced above. This yields the bound

$$
\begin{aligned}
\bar{I}_{N}^{(2)}(i, j) \leqq & \left(\frac{\beta J^{0} K_{1, \lambda}}{N}+\frac{(\beta J)^{2} K_{2, \lambda}}{N}\right) \sum_{k \neq i, j} \bar{I}_{N}^{(2)}(k, j)+\frac{\beta J^{0} K_{3, \lambda}}{N}+\frac{(\beta J)^{2} K_{4, \lambda}}{N} \\
& +O\left(\frac{1}{N^{2}}\right)
\end{aligned}
$$

for some constants $K_{i, \lambda}, i=1,2,3,4$, which are finite, for $\beta$ small enough. This inequality may obviously be iterated indefinitely, and we get

$$
\bar{I}_{N}^{(2)}(i, j) \leqq \frac{\beta J^{0}}{N} A_{1, \lambda}+\frac{(\beta J)^{2}}{N} A_{2, \lambda}+O\left(\frac{1}{N^{2}}\right),
$$

for some constants $A_{1, \lambda}$ and $A_{2, \lambda}$ which are finite, for $\beta$ small enough. This clearly provides the uniform upper bound claimed in (3.13) and proves that

$$
\chi_{N}^{(2)} \leqq \frac{\beta J^{0}}{2} A_{1,1}+\frac{(\beta J)^{2}}{2} A_{2,1}+O\left(\frac{1}{N}\right) .
$$

This inequality proves half of Theorem A, (2) (set $i=2$ ), for $h=0$. To prove (3.25) for $h \neq 0$ one must derive uniform bounds on

$$
N^{-1} \sum_{i=1}^{N} E \mu_{N}\left(\mathbf{S}_{i}\right)^{2} \text { and on } N^{-1} \sum_{1 \leqq i<j \leqq N} E\left(\mu_{N}\left(\mathbf{S}_{i} \cdot \mathbf{S}_{j}\right)^{c}\right)^{2} .
$$

This involves a straightforward extension of the method discussed above which is described in very much detail for related models in the second paper quoted in [1]. There is no point in repeating those arguments here.

From (3.24), (3.12), and (3.4) we derive that

$$
E f\left(\beta H_{N}\right)=f\left(\beta H_{N}^{0}\right)-\frac{\beta J^{2}}{4}+O\left(\frac{1}{N}\right),
$$

in particular,

$$
E f\left(\beta H_{N}\right)=-\frac{\beta J^{2}}{4}+O\left(\frac{1}{N}\right),
$$

for the usual SK model, provided $\beta \geqq 0$ is small enough. This is the first half of Theorem A, (3). In order to complete the proof of Theorem A, (3) we return to Eq. (3.7) for $\varphi\left(\beta H_{N}\right)$. That equation reads

$$
\begin{aligned}
\varphi\left(\beta H_{N}\right)= & \varphi\left(\beta H_{N}^{0}\right)+\left.\frac{\beta}{N^{3 / 2}} \sum_{(i j)} J_{i j} \mu_{N}\left(\mathbf{S}_{i} \cdot \mathbf{S}_{j}\right)\right|_{C_{i j}} \\
& +\frac{\beta}{N^{2}} \sum_{(i j)} \frac{1}{2} J_{i j}^{2}-\left.\frac{\beta}{N^{2}} \sum_{(i j)} \int_{0}^{1} d s_{i j} \int_{0}^{s_{i j}} d s_{i j}^{\prime} J_{i j}^{2} \mu_{N}\left(\mathbf{S}_{i} \cdot \mathbf{S}_{j}\right)^{2}\right|_{C_{i_{j}}^{\prime}},
\end{aligned}
$$


for Ising spins. In analyzing $E \varphi\left(\beta H_{N}\right)^{2}$, the only terms that cannot be calculated explicitly or shown to be $O\left(\frac{1}{N^{2}}\right)$ by using (3.24) are terms proportional to

$$
\left.\left.E J_{i j}^{\alpha_{1}} J_{k l}^{\alpha_{2}} \mu_{N}\left(\mathbf{S}_{i} \cdot \mathbf{S}_{j}\right)^{\delta_{1}}\right|_{C(i j)} \mu_{N}\left(\mathbf{S}_{k} \cdot \mathbf{S}_{l}\right)^{\delta_{2}}\right|_{C(k l)},
$$

where $C(i j)=C_{i j}$, or $=\mathrm{C}_{i j}^{\prime}, \alpha_{p}=1$ or 2 , and $\delta_{p}=1$ or 2 , for $p=1$, 2. Such terms arise in squaring the second or the fourth term on the right-hand side of (3.28), or as cross terms between the second and the fourth term and then taking the expectation. These terms can be analyzed with the help of expansions similar to that in (3.15)-(3.24). The most dangerous terms are

$$
\left.\left.E J_{i j} J_{k l} \mu_{N}\left(\mathbf{S}_{i} \cdot \mathbf{S}_{j}\right)\right|_{C_{i j}} \mu_{N}\left(\mathbf{S}_{k} \cdot \mathbf{S}_{l}\right)\right|_{C_{k l}},
$$

with $(i j) \neq(k l)$. $[$ The terms with $(i j)=(k l)$ can be estimated by using (3.24) and make a total contribution $\left.O\left(\frac{1}{N^{2}}\right).\right]$ We now expand $\left.\mu_{N}\left(\mathbf{S}_{i} \cdot \mathbf{S}_{j}\right)\right|_{C_{i j}}$ to first order in $J_{k l}$ and $\left.\mu_{N}\left(\mathbf{S}_{k} \cdot \mathbf{S}_{l}\right)\right|_{C_{k l}}$ to first order in $J_{i j}$. We then observe by using the Schwarz inequality that what remains to estimate is

$$
\left.\frac{1}{N} E J_{i j}^{2} J_{k l}^{2} \mu_{N}\left(\mathbf{S}_{i} \cdot \mathbf{S}_{j} ; \mathbf{S}_{k} \cdot \mathbf{S}_{l}\right)^{2}\right|_{\mathscr{C}}
$$

for some constraint $\mathscr{C}$. The analysis in the second paper quoted in [1] applies to (3.31) and yields the bound

$$
(3.31) \leqq \frac{\text { const }}{N^{3}}
$$

for $\beta$ sufficiently small.

Remark. If $h \neq 0(3.31) \leqq \frac{\text { const }}{N^{2}}$, and we shall oniy be able to prove that

$$
E\left(\varphi\left(\beta H_{N}\right)-E \varphi\left(\beta H_{N}\right)\right)^{2}=O\left(\frac{1}{N}\right) .
$$

By (3.32) and (3.28), the terms in $E \varphi\left(\beta H_{N}\right)^{2}$ proportional to (3.30) are bounded by

$$
\frac{\beta^{2}}{N^{3}} \cdot \frac{\text { const }}{N^{3}} \sum_{(i j)(k l)} 1 \leqq \frac{\beta^{2} \text { const }}{4 N^{2}},
$$

for $\beta$ small enough.

Other terms of the form (3.29) contributing to $E \varphi\left(\beta H_{N}\right)^{2}-\left(E \varphi\left(\beta H_{N}\right)\right)^{2}$ are dealt with in a similar way. The method of analysis is the one in the second paper quoted in [1]; see also [11]. The proof of (3.32) and of other related estimates is not entirely a trivial matter. However, it follows closely arguments in [1] which we need not reproduce here.

Remark. By using the methods of [1] one can prove estimates

$$
E\left(\varphi\left(\beta H_{N}\right)-E \varphi\left(\beta H_{N}\right)\right)^{2 p}=O\left(\frac{1}{N}\right),
$$

$p=1,2, \ldots$ for general $n$-vector models with $h \geqq 0$, provided $\beta$ is small enough. 
In order to complete the proof of Theorem A, we must finally prove an upper bound on $\chi_{N}^{(1)}$, for $\beta$ small enough. We shall again only consider the simplest case, Ising spins and $h=0$, but the general case can be studied by applying the methods developed in the second paper quoted in [1]; see also [11].

Let

$$
I_{N}^{(1)}(i, j)=\left.\mu_{N}\left(\mathbf{S}_{i} \cdot \mathbf{S}_{j}\right)\right|_{\mathscr{C}} .
$$

The couplings compatible with $\mathscr{C}$ are denoted by $\widetilde{K}_{m n}$, with $\widetilde{K}_{m n}=\frac{\widetilde{J}_{m n}^{0}}{N}+\frac{\widetilde{J}_{m n}}{\sqrt{N}}$, where $\widetilde{J}_{m n}^{0}=a_{0}(m n, \mathscr{C}) J^{0}$ and $\widetilde{J}_{m n}=a(m n, \mathscr{C}) J_{m n}, a_{0}(m n, \mathscr{C}), a(m n, \mathscr{C}) \in[0,1]$. We shall establish an upper bound on

$$
\left|E I_{N}^{(1)}(i, j)\right|,
$$

which is uniform in $i, j, N$ and in the condition $\mathscr{C}$ imposed on the exchange couplings. To this end we apply a Taylor expansion with second order remainder in $\widetilde{K}_{i j}$ to $I_{N}^{(1)}(i, j)$. This yields

$$
\left|E I_{N}^{(1)}(i, j)\right| \leqq\left|E I_{N}^{(1)}(i, j)\right|_{\tilde{K}_{i j}=0} \mid+\frac{\beta J^{0}}{N} a_{1}+\frac{(\beta J)^{2}}{N} a_{2}+O\left(\frac{1}{N^{2}}\right) .
$$

Thus it is enough to show that, for $\beta$ small enough,

$$
\left|E I_{N}^{(1)}(i, j)\right|_{\tilde{K}_{i j}=0} \mid \leqq \operatorname{const} \frac{1}{N},
$$

for a constant independent of $i, j, N$, and $\mathscr{C}$. For symmetric distributions $\mu^{0}(h=0)$ and $E$, we have

$$
\begin{aligned}
& \left.\left|E I_{N}^{(1)}(i, j)\right|_{\tilde{K}_{i j}=0}|=| \beta \sum_{k \neq i, j} \frac{\widetilde{J}_{i k}^{0}}{N} E \partial_{i k} I_{N}^{(1)}(i, j)\right|_{C_{i j k}} \mid \\
& \quad+\left.\beta^{2} \sum_{k \neq i, j} \int_{0}^{1} d s_{i k} \int_{0}^{s_{i k}} d s_{i k}^{\prime} E\left(\frac{\widetilde{J}_{i k}^{0}}{N}+\frac{\widetilde{J}_{i k}}{\sqrt{N}}\right)^{2} \partial_{i k}^{2} I_{N}^{(1)}(i, j)\right|_{\mathscr{C}_{i j k}},
\end{aligned}
$$

with $C_{i j k}, \mathscr{C}_{i j k}$ defined as in (3.17). One verifies easily that, for Ising spins,

$$
\partial_{i k} I_{N}^{(1)}(i, j)=I_{N}^{(1)}(k, j)-I_{N}^{(1)}(i, j) I_{N}^{(1)}(k, i),
$$

and, by definition of $I_{N}^{(2)}$,

$$
\left|I_{N}^{(1)}(i, j) I_{N}^{(1)}(k, i)\right| \leqq \frac{1}{2}\left(I_{N}^{(2)}(i, j)+I_{N}^{(2)}(k, i)\right) .
$$

Hence

$$
\begin{gathered}
\left.\left|\beta \sum_{k \neq i, j} \frac{\widetilde{J}_{i k}^{0}}{N} E \partial_{i k} I_{N}^{(1)}(i, j)\right|_{C_{\imath j k}}\left|\leqq \frac{\beta J^{0}}{N}\right| \sum_{k \neq i, j} E I_{N}^{(1)}(k, j)\right|_{C_{i j k}} \mid \\
+\left.\frac{\beta J^{0}}{N}\right|_{k \neq i, j}\left(\left.E I_{N}^{(2)}(i, j)\right|_{C_{i j k}}+\left.E I_{N}^{(2)}(k, i)\right|_{C_{i j k}}\right) \mid .
\end{gathered}
$$
The second term on the right-hand side of (3.40) is bounded above by $\frac{\beta^{2} \text { const }}{N}$; see
(3.24). 
Next, we study the second term on the right-hand side of (3.37). For Ising spins, we have

$$
\partial_{i k}^{2} I_{N}^{(1)}(i, j)=-2 I_{N}^{(1)}(k, j) I_{N}^{(1)}(i, k)+2 I_{N}^{(1)}(i, j)\left(I_{N}^{(1)}(i, k)\right)^{2} .
$$

The right-hand side of (3.41) is bounded from above in terms of $I_{N}^{(2)}$ by using inequalities like (3.39). The resulting upper bound is further estimated by using (3.24). By (3.35) and (3.40), this yields the bound

$$
\left|E I_{N}^{(1)}(i, j)\right| \leqq \frac{\beta J^{0} c_{1}+(\beta J)^{2} c_{2}}{N}+\frac{\beta J^{0}}{N} \sum_{k \neq i, j}\left|E I_{N}^{(1)}(k, j)\right|_{C_{i j k}} \mid,
$$

where the constants $c_{1}$ and $c_{2}$ are finite and independent of $i, j, N$, and $\mathscr{C}$, for $\beta$ small enough. Inequality (3.42) can be iterated, and we obtain

$$
\bar{I}_{N}^{(1)}(i, j) \leqq \frac{\beta J^{0} c_{1}^{\prime}+(\beta J)^{2} c_{2}^{\prime}}{N}
$$

where

$$
\bar{I}_{N}^{(1)}(i, j)=\sup _{\mathscr{C}}\left|E \mu_{N}\left(\mathbf{S}_{i} \cdot \mathbf{S}_{j}\right)\right|_{\mathscr{C}} \mid,
$$

and $c_{1}^{\prime}, c_{2}^{\prime}$ are finite constants independent of $i, j$, and $N$, for $\beta$ small enough.

Inequality (3.43) proves Theorem $\mathrm{A},(2)$, for $i=1$. This completes our proof of Theorem A, for Ising spins and with $h=0$. The extension of Theorem A, (1) and (2) to general $n$-vector spins and $h \neq 0$ is quite straightforward, given the techniques in Sect. 3 of the second paper quoted in [1]; see also [11].

Next, we turn to a sketch of the proof of Theorem B. Our starting point is the Taylor expansion of the free energy with second order remainder given in (3.7), but for the models introduced in (2.8), (2.9).

Let $H_{A}^{\gamma}$ be as in (2.8), (2.9), and define

$$
\stackrel{\circ}{H}_{\Lambda}^{\gamma}=-\sum_{(i j) \subset \Lambda} \tilde{J}_{i j}^{0} S_{i} S_{j}-h \sum_{i \in \Lambda} S_{i},
$$

where $S_{i}= \pm 1$ are Ising spins, and

$$
\widetilde{J}_{i j}^{0}=\widetilde{J}^{0} \gamma^{d} \exp [-\gamma|i-j|],
$$

see (2.9). We define $\varphi\left(\beta H_{A}\right)$ by

$$
\varphi\left(\beta H_{\Lambda}\right)=\frac{1}{|\Lambda|} \log \mu^{0}\left(e^{-\beta H_{\Lambda}}\right) .
$$

As in (3.7), successive Taylor expansions in

$$
J_{i j}^{\gamma} \equiv \mathscr{J}_{i j} \gamma^{d / 2} \exp [-\gamma|i-j|],
$$

see (2.9), with second order remainders yield the identity

$$
\begin{aligned}
\varphi\left(\beta H_{\Lambda}^{\gamma}\right)=\varphi\left(\beta H_{\Lambda}^{\gamma}\right) & +\left.\frac{\beta}{|\Lambda|} \sum_{(i j) \subset \Lambda} J_{i j}^{\gamma} \mu_{\Lambda}^{\gamma}\left(S_{i} S_{j}\right)\right|_{C_{i j}} \\
& +\left.\frac{\beta^{2}}{|\Lambda|} \sum_{(i j) \subset \Lambda} \int_{0}^{1} d s_{i j} \int_{0}^{s_{i j}} d s_{i j}^{\prime}\left(J_{i j}^{\gamma}\right)^{2} \mu_{\Lambda}^{\gamma}\left(S_{i} S_{j} ; S_{i} S_{j}\right)\right|_{C_{i j}^{\prime}},
\end{aligned}
$$

with $C_{i j}$ and $C_{i j}^{\prime}$ as in (3.7). 
Equation (3.44) and the result of [1] yield an analogue of the last part of Theorem A, (3). More precisely, one can show that, for all $\gamma>0$,

$$
\lim _{\Lambda>\mathbb{Z}^{d}} \varphi\left(\beta H_{\Lambda}^{\gamma}\right)=\lim _{\Lambda>\mathbb{Z}^{d}} \tilde{E} \varphi\left(\beta H_{\Lambda}^{\gamma}\right)
$$

in the sense that $\varphi\left(\beta H_{\Lambda}\right)-\widetilde{E} \varphi\left(\beta H_{\Lambda}\right) \rightarrow 0$, as $\Lambda \nearrow \mathbb{Z}^{d}$, in $L_{p}$ with respect to $\widetilde{E}$, for all $p$ with $1 \leqq p<\infty$. It is therefore enough to study the behaviour of $\lim _{A \times \mathbb{Z}^{d}} \tilde{E} \varphi\left(\beta H_{A}\right)$, as $\gamma \searrow 0$, in order to prove Theorem B.

In taking the expectation $\widetilde{E}$ of both sides of Eq. (3.44) we use the fact that

$$
\left.\widetilde{E} J_{i j}^{\gamma} \mu_{\Lambda}^{\gamma}\left(S_{i} S_{j}\right)\right|_{C_{i_{J}}}=0 \text {, }
$$

since $\widetilde{E} J_{i j}^{\gamma}=0$ and $\left.\mu_{\Lambda}^{\gamma}\left(S_{i} S_{j}\right)\right|_{C_{i j}}$ is independent of $J_{i j}^{\gamma}$, by the definition of $C_{i j}$. We then obtain

$$
\tilde{E} \varphi\left(\beta H_{\Lambda}^{\gamma}\right)=\varphi\left(\beta \dot{H}_{\Lambda}^{\gamma}\right)+\frac{\beta^{2}}{|\Lambda|} \sum_{(i j) \subset \Lambda} \int_{0}^{1} d s_{i j} \int_{0}^{s_{1 j}} d s_{i j}^{\prime} \tilde{E}\left(J_{i j}^{\gamma}\right)^{2}\left[1-\left.\mu_{\Lambda}^{\gamma}\left(S_{i} S_{j}\right)^{2}\right|_{C_{i j}^{\prime}}\right] .
$$

We have used that $\left(S_{i} S_{j}\right)^{2}=1$, i.e.

$$
\mu_{\Lambda}^{\gamma}\left(S_{i} S_{j}\right)^{2}=1
$$

but our analysis could be extended to general $n$-vector spins. Next, we note that

$$
\tilde{E}\left(J_{i j}^{\gamma}\right)^{2}=\widetilde{J}^{2} \gamma^{d} \exp [-2 \gamma|i-j|] .
$$

Moreover, the results in [1] show that if $-\beta_{0}<\beta<\beta_{0}$, for some $\beta_{0}>0$ independent of $\gamma$,

$$
\left.\widetilde{E}\left(J_{i j}^{\gamma}\right)^{2} \mu_{\Lambda}^{\gamma}\left(S_{i} ; S_{j}\right)^{2}\right|_{C} \leqq K \widetilde{J}^{4} \gamma^{2 d} \exp [-4 \gamma|i-j|],
$$

for some finite constant $K$. The point is that $\beta_{0}$ and $K$ are independent of $\gamma$. This may not be obvious to the reader, but follows readily from [1] and the bound

$$
\sum_{j \neq 0} \gamma^{d} \exp [-2 \gamma|j|] \leqq b<\infty,
$$

uniformly in $\gamma>0$. In fact, the bound (3.48) holds for general $n$-vector spins and an arbitrary magnetic field $h$. [Of course,

$$
\left.\mu_{\Lambda}^{\gamma}\left(S_{i} ; S_{j}\right)=\mu_{\Lambda}^{\gamma}\left(S_{i} S_{j}\right), \quad \text { for } \quad h=0 .\right]
$$

Hence

$$
\begin{aligned}
\widetilde{E} \varphi\left(\beta H_{\Lambda}^{\gamma}\right)= & \varphi\left(\beta \dot{H}_{\Lambda}^{\gamma}\right)+\frac{\beta^{2} \widetilde{J}^{2}}{2|\Lambda|} \sum_{(i j) \subset \Lambda} \gamma^{d} \exp [-2 \gamma|i-j|] \\
& -\frac{\beta}{2|\Lambda|} K \widetilde{J}^{4} \sum_{(i j) \subset \Lambda} \gamma^{2 d} \exp [-4 \gamma|i-j|] .
\end{aligned}
$$

Clearly

$$
\lim _{\Lambda \rightarrow \mathbb{Z}^{d}} \frac{1}{|\Lambda|} \sum_{(i j) \subset \Lambda} \gamma^{d} \exp [-2 \gamma|i-j|]=\frac{1}{2} \sum_{0 \neq j \in \mathbb{Z}^{d}} \gamma^{d}[\exp -2 \gamma|j|] \equiv \frac{1}{2} b_{\gamma} \leqq \frac{1}{2} b<\infty,
$$

for all $\gamma>0$. Moreover,

$$
\lim _{\gamma>0} b_{\gamma}=\int e^{-2|x|} d^{d} x \equiv b_{0}
$$


and

$$
\frac{1}{|\Lambda|} \sum_{(i j) \subset \Lambda} \gamma^{2 d} \exp [-4 \gamma|i-j|] \leqq \frac{1}{2} \sum_{0 \neq j \in \mathbb{Z}^{d}} \gamma^{2 d} \exp [-2 \gamma|i-j|] \leqq b \gamma^{d} .
$$

Hence

$$
\lim _{\Lambda \rightarrow \mathbb{Z}^{d}} \tilde{E} \varphi\left(\beta H_{\Lambda}^{\gamma}\right)=\lim _{\Lambda \rightarrow \mathbb{Z}^{d}} \varphi\left(\beta \dot{H}_{\Lambda}^{\gamma}\right)+\frac{\beta \tilde{J}^{2}}{4} b_{\gamma}-O\left(\gamma^{d}\right)
$$

It is easy to show, using the techniques developed above (see also [1, 11]), that

$$
\lim _{\Lambda \rightarrow \mathbb{Z}^{d}} \varphi\left(\beta \dot{H}_{\Lambda}^{\gamma}\right)=\lim _{N \rightarrow \infty} \varphi\left(\beta H_{N}^{0}\right)+O\left(\gamma^{d}\right),
$$

for a suitable choice of $\tilde{J}^{0}$. Hence

$$
\lim _{\gamma>0}\left(\lim _{\Lambda>\mathbb{Z}^{d}} f_{\Lambda}(\beta, h=0, \gamma)\right)=\lim _{N \rightarrow \infty} f\left(\beta H_{N}^{0}\right)-\frac{\beta \tilde{J}^{2}}{4} b_{0}=\lim _{N \rightarrow \infty} f\left(\beta H_{N}^{0}\right)-\frac{\beta J^{2}}{4},
$$

if $\tilde{J}$ is chosen such that $\tilde{J}^{2} b_{0}=J^{2}$.

This completes our proof of Theorem B.

Remark. It should be emphasized that the techniques developed in this paper and in [1] enable us to prove much more general results relating the short-range models to the mean-field models, as $\gamma \searrow 0$. We can prove an analogue of Theorem B for general $n$-vector models with $h \neq 0$ and have results concerning the susceptibilities $\chi^{(1)}, \chi^{(2)}$, etc.

\section{Conclusions}

In this paper we have given a fairly detailed analysis of the high-temperature behaviour of general mean-field spin glass models, including the SK-model. The spins can be $n$-vectors, $n=1,2,3, \ldots$, and the magnetic field $h$ need not vanish for our techniques to be applicable. Furthermore, we have related the mean-field models to short-range spin glasses in the limit where the range of the exchange couplings tends to $\infty$, in a way specified in (2.9).

For the SK-model with $h=0$, our results suffice to conclude the existence of a phase transition as the temperature is lowered, as pointed out already in [10,2]. In situations where the techniques of [2] apply they give more precise information and better bounds on the transition temperature than ours, but their scope appears to be considerably more limited.

Acknowledgements. We thank D. Ruelle for a copy of [2] and for describing their results to us. J.F. thanks the I.H.E.S. for hospitality during the period when this paper was completed.

\section{References}

1. Fröhlich, J., Zegarlinski, B.: The disordered phase of long-range Ising spin glasses. Europhys. Lett. 2, 53 (1986)

Fröhlich, J., Zegarlinski, B.: The high-temperature phase of long-range spin glasses. Commun. Math. Phys. 110, 121 (1987) 
2. Aizenman, M., Lebowitz, J.L., Ruelle, D.: Some rigorous results on the SherringtonKirkpatrick spin glass model. Commun. Math. Phys. 112, 3-20 (1987)

3. Parisi, G.: A sequence of approximated solutions to the SK model for spin glasses. J. Phys. A 13, L 115. The order parameter for spin glasses: A function on the interval 0-1. A 13, 1101. Magnetic properties of spin glasses in a new mean field theory. A 13, 1887 (1980)

Mézard, M., Parisi, G., Virasoro, M.: SK model: The replica solution without replicas. Europhys. Lett. 1, 77 (1986)

4. Thouless, D.J.: Phys. Rev. Lett. 56, 1082 (1986)

5. Chayes, J.T., Chayes, L., Sethna, J.P., Thouless, D.J.: A mean field spin glass with short-range interactions. Commun. Math. Phys. 106, 41-89 (1986)

6. Edwards, S.F., Anderson, P.W.: J. Phys. F 5, 965 (1975)

7. Bray, A.J., Moore, M.A.: Lower critical dimension of Ising spin glasses: A numerical study. J. Phys. C17, L463 (1984); Critical behavior of the three-dimensional Ising spin glass. Phys. Rev. B 31, 631 (1985). Proceedings of the 1986 Heidelberg Colloquium on "Glassy Dynamics and Optimization." Lecture Notes in Physics. Berlin, Heidelberg, New York: Springer

8. Bovier, A., Fröhlich, J.: A heuristic theory of the spin glass phase. J. Stat. Phys. 44, 347 (1986)

9. Fisher, D., Huse, D.: Preprints, AT \& T Bell Laboratories, 1986

10. Sherrington, D., Kirkpatrick, S.: Solvable model of a spin glass. Phys. Rev. Lett. 35, 1792 (1975)

11. Fröhlich, J., Zegarlinski, B.: Connections between mean-field spin glasses and short-range spin glasses in the infinite range limit (unpublished)

Communicated by A. Jaffe

Received June 22, 1987 